\title{
Modelling of the simple pendulum Experiment
}

\author{
L.Palka ${ }^{1}$, F. Schauer ${ }^{2}$ and P. Dostal ${ }^{1}$ \\ ${ }^{1}$ Tomas Bata University in Zlín, Zlín, Czech Republic \\ ${ }^{2}$ Trnava University in Trnava, Slovak Republic
}

\begin{abstract}
Abstract - work focuses on the design of the simulation embedded in remote experiment "Simple pendulum " built on the Internet School Experimental System (ISES). This platform is intended for wide educational purposes at schools and universities in order to provide the suitable measuring environment for students using conventional computing resources Informatics.
\end{abstract}

\section{Introduction}

The modern trend in scientific and teaching experimentation are the remote e-laboratories and estores for their HW and SW components[2,4]. For the purpose of management of remote laboratories the interconnected datacentres, creating the seed of the integrated net of remote laboratories for EU programme is being built [5]. Why do we deal with the matter?

Traditional teaching methods for students at schools and universities are outdated and not effective enough. Students often expect faster and more understandable teaching methods in the field of physics, biology, chemistry and electro-engineering, which can help them to better perceive real world phenomena. The problem is also an accessibility of the educational materials, especially for distant students who nowadays prefer studying scientific topics using their computers via the Internet. These problematic points are effectively solved by the remote laboratories (RLs) so called e-laboratories. The RLs built on the ISES platform are developed longer time by the RL Consortium consisted of three universities (Charles University in Prague, Tomas Bata University in Zlín and Trnava University in Trnava) for educational purposes. The ISES is an advanced tool deployed for real-time operation, data acquisition, data processing, hardware $(\mathrm{HW})$ controlling and visualizing.

The platform is an open system consisting of the ISES hardware and software components intended for hands-on experiments called ISES WIN. However, there is also an alternative for using remote experiments (REs), the ISES WEB Control Kit. Both types of the experiments are built as the burst (fast) and normal (slow) to offer students a wider spectrum of knowledge. The initial version of ISES RLs has been developed by Charles University in Prague. When the ISES RL became the time-proven educational tool, it was significantly improved to a higher level tool by Tomas Bata University in Zlín in cooperation with Charles University in Prague. They implemented features

${ }^{\mathrm{a}}$ Corresponding author: lukas.palka@email.cz for the new user environment, EASY REMOTE-ISES, in order to simply building REs by laymen [4][16].

Let us describe the ISES RL concept. It consists of six units as the HW components (convertor, control board, physical modules categorized as meters, sensors and devices), Measureserver, Imageserver, Webserver, Datawarehouse and Webclient. More technical details and deployed applications are available in [1][2][3][4][6] and [7]. All the ISES RLs were recently integrated into a new platform so called Remote Laboratory Management System (REMLABNET). It provides autonomous units as services to the ISES RL administrators and clients. The schematic arrangement of all the involved units and corresponding communication is presented in Figure B.

The layout of the paper is following. In Chapter 1, the typical scheme of the communication of a typical remote experiment (RE), built as the finite-state machine (FSM) [2], using the Datawarehouse and Internet School Experimental System (ISES) physical hardware, is described[10] (for the ease of reading we will next denote the set of the individual remote experiment by the word rig). Also, the control program compiling and the type of the data generated and transferred is shortly described. More details may be found in corresponding literature [2][3]

The Chapter 2 is devoted to describing the architecture Remote Experiments and the corresponding integrating management system, called for our purposes Remote Laboratory Management System (RLMS) [10]. The Chapter 3 is devoted to the actual communication design and database scheme of remote laboratories. The Chapter 4 is then focused on the simulation of a simple pendulum in remote experiments linked to RemLabNet environment. The chapter 5 is oriented on acquainting the reader with the results of a simulation of a simple pendulum in remote experiments linked to RemLabNet environment. The final chapter 6 is focused on the use of 
data mining from simple pendulum, use in RemLabNet projection system and the results on the portal.

\section{State of the art}

Only recently has emerged a serious problem stemming from analysis of research data. ISES is a powerful tool for process and experiments control, acquisition, collecting and data processing in real time. Let us mention the basic features of the ISES system, more detailed description may be found elsewhere [2][10]. The basis of the system is ISES board, which is available in several versions, differing depending on the number of inputs/outputs and also on type of communication with the control PC (by PCI card, USB connector, Wi-Fi). To this board are, by a unique connector, plugged in sensors like: ammeter, voltmeter, thermometer, position sensor, ohmmeter, load cell, anemometer, microphones, sonar, light gate, $\mathrm{pH}$ meter, conductivity meter, heart rate monitor, etc. [8]. The layout arrangement of the RE is in Figure A.

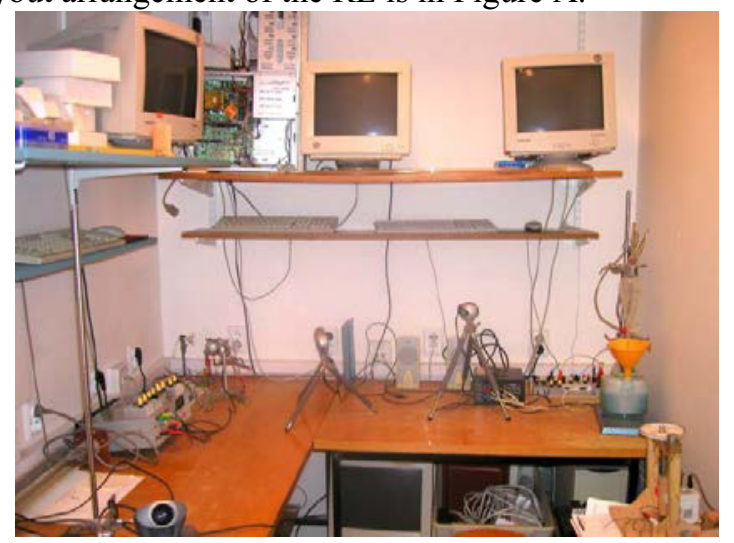

Figure A. ISES - Internet School Experimental System elaboratory in MFF UK in Prague.

The most important component is the Measureserver module, functioning as finite-state machine (FSM) controlled by the controlling program of the PSC script file. The main feature of the Measureserver, is to communicate with the physical hardware and to check the setup of the ISES panel and its sensors/meters and to take care about their data collection and processing. Other parts of the system are ImageServer for life view of the remote experiment, Web server for the communication between RE and the client. Also, aprt of the RE is the communication web page as the interface communicating with the RE over the Internet by the client.

The inevitable part of the RE system is the data warehouse for the storage of data for all above systems. It is a centralized repository service to Measureserver, web server, image server and other components of the solution.

In this article we will discuss this last part of the system with respect of data security, but not only from the perspective a single RE, but of the whole RLMS. The layout arrangement of the RE is in Figure B.

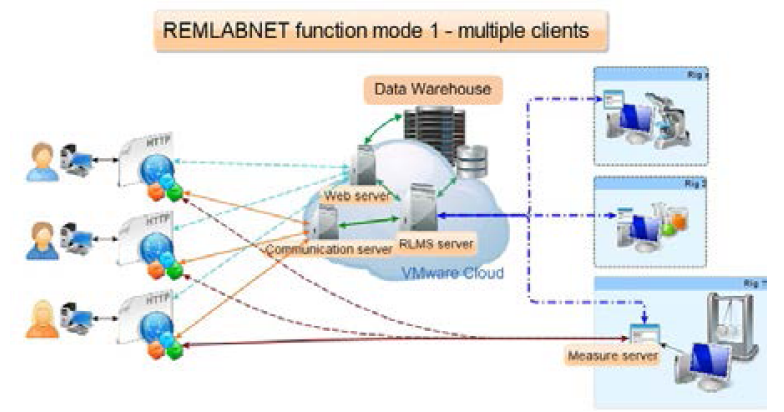

Figure B. REMLABNET function mode 1 - multiple clients.

A serious problem stemming from security aspects of e-laboratories has emerged only recently [19]. Let us describe first the data generated and that are processed in every ISES rig working on the communication principle server-client and the functioning of the superordinate Remote Laboratory management system (RLMS). The controlling of every rig by client is enabled via Web interface, by means of which the user can perform the appropriate settings, options, and starting or stopping of the remote experiment (RE).

The measured data from the experiment delivered from the MeasureServer are stored in the data storage . RLMS is a system for a database-driven Web application. This is seen from the figure 2, where LMS is divided terms of safety in three parts. Database-driven Web applications are very common in today's Web-enabled society. LMS consist of a back-end database with Web pages that contain server-side script written in a programming language that is capable of extracting specific information from a database depending on various dynamic interactions with the user.

Remote experiments problematic is the topic of scientific activities of the group since 2005, when the first remote experiment started to be built. We relied on the enormous know-how of Assoc. Prof. F. Lustig from Department of Physics Education of Faculty of Mathematics and Physics, Charles University in Prague, where the universal and very useful modular computer oriented set Internet School Experimental System (ISES) was designed at the beginning of 90th [21].

\section{Phenomen simulation concept}

The project deals with classical simple pendulum with adjustable amplitude and low damping, enabling experiments with duration of app. till 1 hour. The output is in form of the instantaneous angle of deflection (red curve in Figure A 1.2) on time and the counter of number of swings (blue marks). All data are available for downloading.

In the description of the use of the system a RemLabNet is the remote experiment simple pendulum with multipurpose use, starting from the measurement of acceleration of gravity, kinematics (instantaneous velocity and acceleration with its tangential and normal components), dynamics (acting forces, equation of motion), and energy (potential and kinetic energy, energy 
conservation law, work of dissipative forces) of curvilinear motion. Experiment is suitable as a demonstration experiment in the lectures, in the seminars as the data source for calculations and in the laboratory exercises.

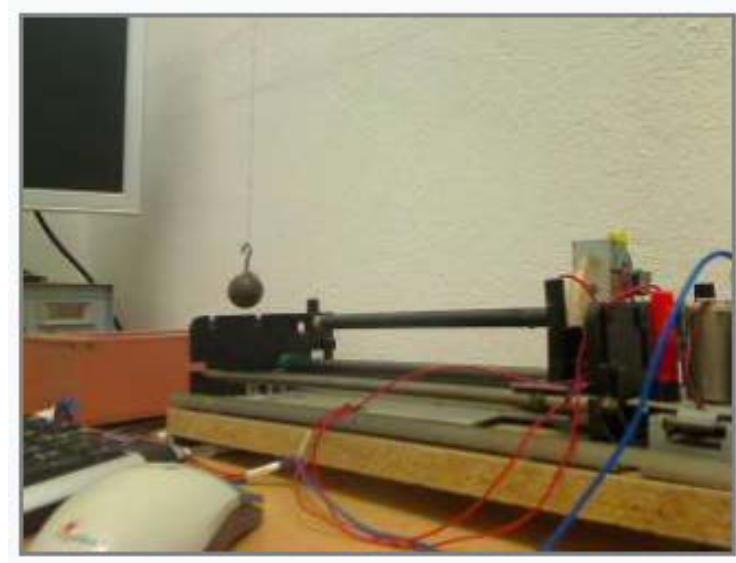

Figure C. RemLabNet simple pendulum experiment.

The following figure shows a simple pendulum, where you can see a workable solution in practice in the lab.

The following illustration shows the description and amplitudes during the course of a simple pendulum. They are seen here with runs pendulum course of its amplitude and data that were generated by the pendulum. Public version of the data is in Chapter VII.

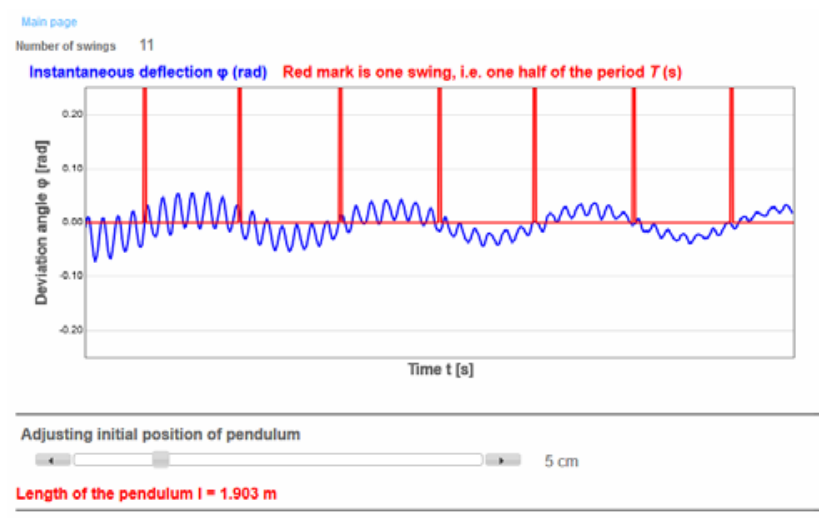

Figure D. amplitude easy course pendulum.

\section{Simple pendulum model in easy java simulations - Theory}

Classical simple pendulum with adjustable amplitude and low damping, enabling experiments with duration of app. till 1 hour. The output is in form of the instantaneous angle of deflection (red curve in Figure A 1.2) on time and the counter of number of swings (blue marks). All data are available for downloading.

Description of the use:

The remote experiment simple pendulum with multipurpose use, starting from the measurement of acceleration of gravity, kinematics (instantaneous velocity and acceleration with its tangential and normal components), dynamics (acting forces, equation of motion), and energy (potential and kinetic energy, energy conservation law, work of dissipative forces) of curvilinear motion. Experiment is suitable as a demonstration experiment in the lectures, in the seminars as the data source for calculations and in the laboratory exercises.

We simulate the motion of a pendulum which has a fixed obstacle in the trajectory of its arm. For simplicity, we assume the obstacle is aligned vertically with the turning point of the pendulum. See the figure above.

The resulting motion can be considered as the superposition, to each side of the obstacle, of the motion of tow pendula, each with a different arm length. Hence, the differential equation of the interrupted pendulum is just:

$$
\begin{aligned}
& \frac{d \cdot \theta}{d t}=\omega \\
& \frac{d \cdot \omega}{d t}=-\frac{g}{l} \sin (\theta)
\end{aligned}
$$

if only the value of 1 is different when the pendulum is at each side of the obstacle. Moreover, the collision of the pendulum's arm with the obstacle produces a sudden change of the angular velocity of the pendulum (while the linear velocity of the pendulum bob remains does not change as result of the collision). We will treat this collision by means of an event.

\section{Theoretical background kinematics the curvilinear motion}

Equations should be centred and should be numbered with the number on the right-hand side.

The velocity of a curvilinear motion is defined

$\mathrm{v}=\frac{\mathbf{d} \mathbf{r}}{\mathbf{d} t}$,

where $\boldsymbol{r}$ is the position vector. The direction of the velocity $\boldsymbol{v}$ is given by the tangent to the trajectory ( Figure 1).

The basic property of any curvilinear motion (even those with constant velocity) is its non zero normal acceleration $\boldsymbol{a}_{\mathrm{n}}$. It is due to the fact that the direction of the unit vector of the velocity is not constant and changes with time.

$\mathbf{a}_{\mathbf{n}}=\frac{\mathrm{d}\left(\frac{v}{v}\right)}{\mathbf{d} t}$

Its value is

$\mathbf{a}_{\mathbf{n}}=\frac{v^{2}}{R}$, 
Where $v$ is the value of the instantaneous velocity and $R$ is the radius of the curvature of the trajectory in and its direction is the normal to the trajectory.

If the motion has variable velocity, then we introduce, similarly as with linear motion, the tangential acceleration

$\mathbf{a}_{\mathbf{t}}=\frac{d v}{d t}$.

With the direction given by the tangent to the trajectory.

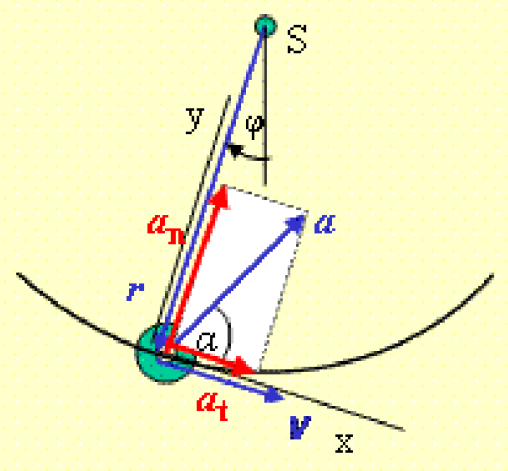

Figure 1. Tangential and normal acceleration of the curvilinear motion (the angle of deflection in the figure is ?<0)

To study the curvilinear motion we will use the simple pendulum (Figure 1). It is a weight of the mass $m$, hanging on the suspension of the length $r$. The pendulum is moving under the influence of the force of weight $\boldsymbol{G}$ by the harmonic motion, described by the angular displacement? $(><0$ !)

$\varphi=\varphi_{0} \sin (\Omega)$

where $?_{o}$ is its amplitude and? is the angular frequency

$\mathrm{g}=2 \%=\frac{2 \pi}{T}=\sqrt{\frac{r}{g}}$,

to the weight.

For the normal acceleration we can then write, using eq.(5), ( Figure 2)

$a_{n}=\mathbf{r} \omega^{2}=r\left(\frac{\mathbf{d} \varphi}{\mathbf{d} t}\right)^{2}=r\left(\varphi_{0} \Omega \cos \Omega t\right)^{2}$

where $r$ is the radius vector value and $\quad \sigma=\frac{d \varphi}{d t}$ is the angular velocity of the pendulum motion.

For the tangential acceleration, using the angular

acceleration $E=\frac{d^{2} \varphi}{d t^{2}}$, we have

$a_{t}=\mathbf{r} z=r\left(\frac{\mathbf{d}^{2} \phi}{\mathbf{d} t^{2}}\right)=-r\left(\phi Q^{2} \sin \Omega\right)$



Figure 2. Tangential and normal acceleration of the simple pendulum, eq.(6) and (7);

$$
r=2 \mathrm{~m}, ?_{o}=0,1 \operatorname{rad} T=3 \mathrm{~s}, \mathrm{~m}=0,1 \mathrm{~kg}
$$

\section{Dynamics of the curvilinear motion}

Why is the simple pendulum moving according to eq. (5)? The equation of motion (2. Newton' law of motion), expressing the action of forces from other bodies on the motion of a body is

$$
\sum_{i} \mathrm{~F}_{i}=m \mathrm{a} \text {. }
$$

where $\boldsymbol{a}$ is the acceleration of the body. For a simple pendulum from Figure 3 the eq.(8) reads for the $\mathrm{x}$ axis (?

$$
G \sin (\varphi)=-m \alpha_{t}
$$

whereas for the y axis

$$
T-G \cos (\varphi)=m \alpha_{n} \text {. }
$$

Equation (9) is the differential equation for the angular displacement? (where we used eq.(6))

$$
\left(\frac{\mathbf{d}^{2} \varphi}{\mathrm{d} t^{2}}\right)=-\frac{g}{r} \sin (\varphi) \text {. }
$$

The eq.(11) is analytically not soluble, but for small angular displacements (??) =? and the solution is (see eq. (5))

$$
\varphi=\varphi_{0} \sin (\Omega)
$$

Then we can write, using eqs. (5) and (6) for the pull $T$ in the suspension of the pendulum the important for the measurement expression ( see Figure 3 and its dependence in Figure 4 )

$$
T=m g \cos \left[\varphi_{0} \sin (\Omega)\right]+m r(\varphi \Omega \cos \Omega)^{2} \text {. }
$$




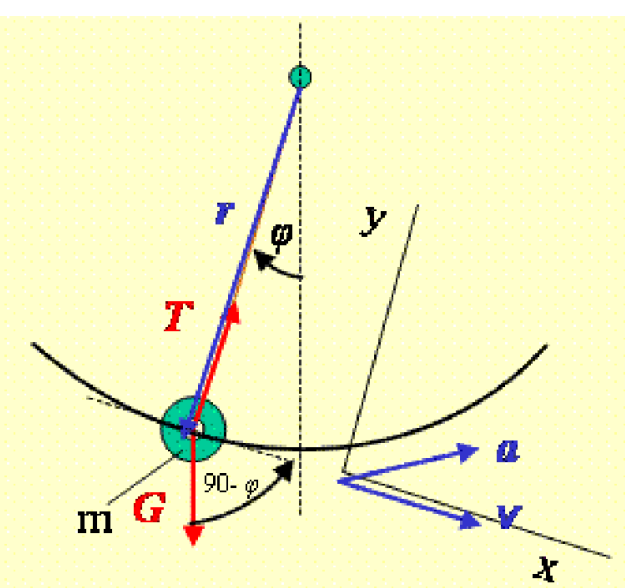

Figure 3. Dynamics of the simple pendulum motion



Figure 4. Time dependence of the pull T in the suspension of the pendulum, eq.12; $r=2 m, ?_{o}=0,1 \mathrm{rad} T=3 \mathrm{~s}, m=0,1 \mathrm{~kg}$

\section{Energy of the curvilinear motion}

The kinetic energy of the pendulum is, using eq. (5) and the definition of the angular velocity

$$
E_{k}=\frac{1}{2} m v^{2}=\frac{1}{2} m(r \omega)^{2}=\frac{1}{2} m\left[r \varphi_{0} \Omega \cos (\Omega t)\right]^{2} \text {. }
$$

The potential gravitation energy of the weight of the pendulum is, using the expression for the pendulum position $h$ above the reference level (taken as the lowest point of the pendulum swing) $h=l(1-\cos ?(t))$ ( Figure 5)

$$
E_{y}=m g h=m g r[1-\cos (\phi(t))]=m g r\left(1-\cos \left[\varphi_{0} \sin (\Omega)\right]\right\}
$$

For the pendulum motion the law of the conservation of the mechanical energy is fulfilled (Figure 6)

$$
E_{k 1}+E_{y \text { weightl }}=E_{k 2}+E_{y} \text { weight }
$$

\section{Simple pendulum model in easy java simulations - results}

\section{Motion of a Pendulum}

This simulation models the motion of a simple pendulum. You can change the mass of the pendulum bob, the length of the string, the acceleration due to gravity, and the initial deflection of the pendulum. You then can observe the resulting oscillations of the pendulum, as well as see bar graphs of the kinetic, potential and total mechanical energy and plots of the pendulum's angular position, angular speed, and angular acceleration as a function of time.

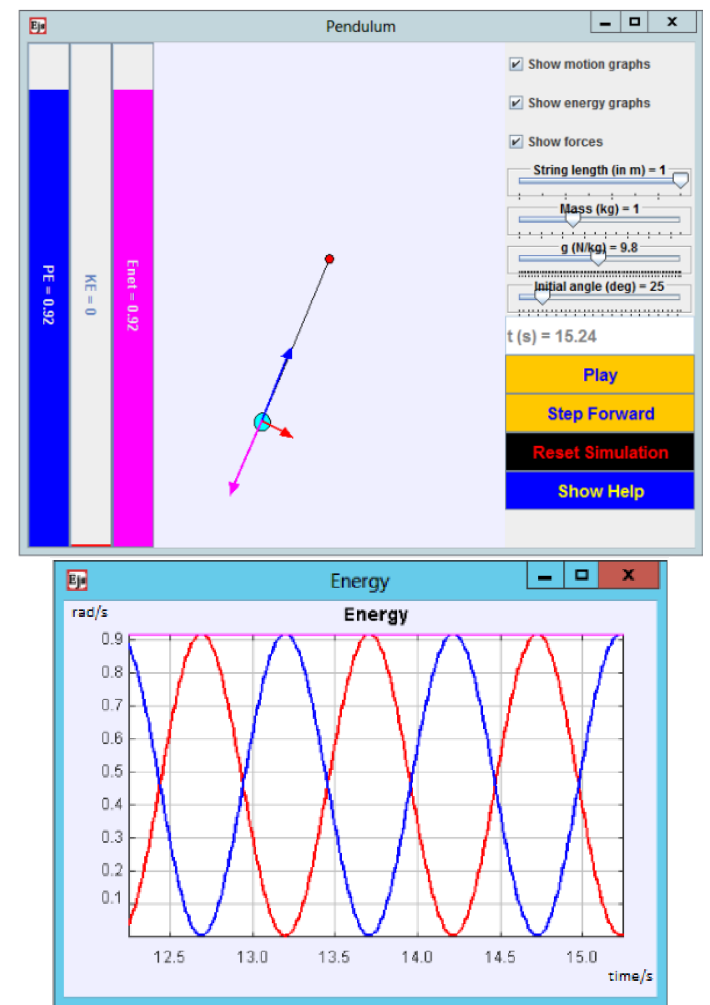

Figure D. Controlling the environment of easy java simulation and output to EJ.

Let us describe individual phases of the knowledge mining process in more detail.

Model:

Variables

We formulate the following basic set of variables:

- $t$ - the time, $h$ - the increment for the time at each evolution step, and $g$ the acceleration due to gravity.

- $m$ - the mass of the pendulum bob, lTotal -the full length of the pendulum, and $d$ - the distance from the turning point to the obstacle. ?

- leftSide is a boolean flag that indicates whether the pendulum is to the left side of the obstacle.

- $\quad l$ is the current value of the pendulum length to be used in the differential equation.

- $\quad$ theta and omega are the angular magnitudes.

- Finally, $x 1$ and $y 1$ are the Cartesian coordinates of the folding point of the (broken) pendulum, and $x 2$ and $y 2$ are the coordinates of the position of the pendulum bob.

The table of variables is then: 


\begin{tabular}{|c|c|c|c|c|}
\hline \multicolumn{3}{|c|}{ 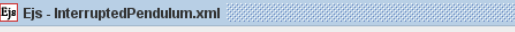 } & \multicolumn{2}{|c|}{$a^{2} \square^{7}$ 区 } \\
\hline \multicolumn{3}{|c|}{ Description Model View } & \multirow[b]{2}{*}{ Custom } & 漛 \\
\hline \multirow{2}{*}{$\begin{array}{l}\text { - Variables } \\
\text { Variables } \\
\text { Name }\end{array}$} & Initialization Evolution & Constraints & & $\square$ \\
\hline & Value & Type & Dimension & 而 \\
\hline and & 0 & double & & \\
\hline $\mathrm{h}$ & 0.1 & double & & [橉] \\
\hline$g$ & 9.8 & double & & \\
\hline m & 1 & double & & $\triangleright$ \\
\hline 1Total & 1.8 & double & & b \\
\hline $\mathrm{d}$ & 1.2 & double & & \\
\hline leftside & false & boolean & & 画 \\
\hline 1 & ITotal & double & & [i] \\
\hline theta & Math.PI/4 & double & & \\
\hline omega & 0 & double & & \\
\hline $\mathrm{x} 1$ & $d *$ Math.sin (theta) & double & & \\
\hline $\mathrm{y}^{1}$ & - $d *$ Math.cos (theta) & double & & \\
\hline $\mathrm{x} 2$ & $\times 1+($ Total-d $) *$ Math.sin $($ theta $)$ & a) double & & \\
\hline $\mathrm{y}^{2}$ & y1 $-($ ITotal-d $) *$ Math.cos (theta) & a) double & & \\
\hline
\end{tabular}

\section{Initialization}

The variables have been correctly initialized in the table of variables.

\section{Evolution}

The evolution needs a page of ordinary differential equations (ODE) with an event. The ODE editor looks as in the figure:

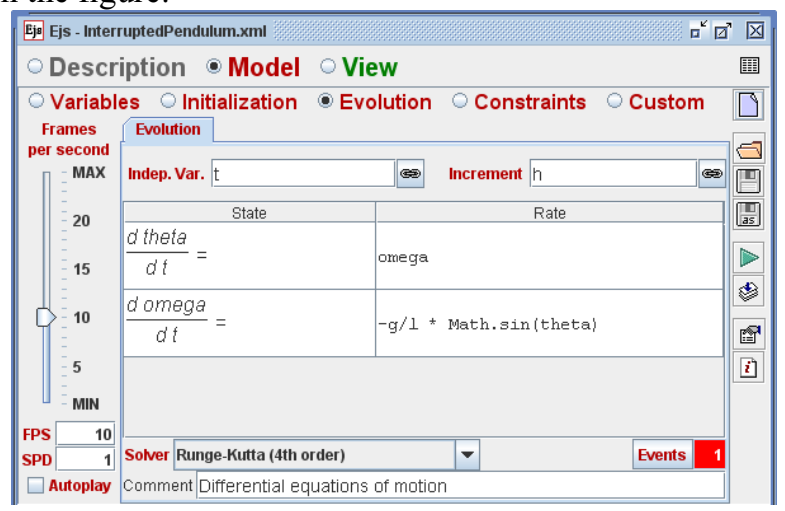

The event is defined by the event page:

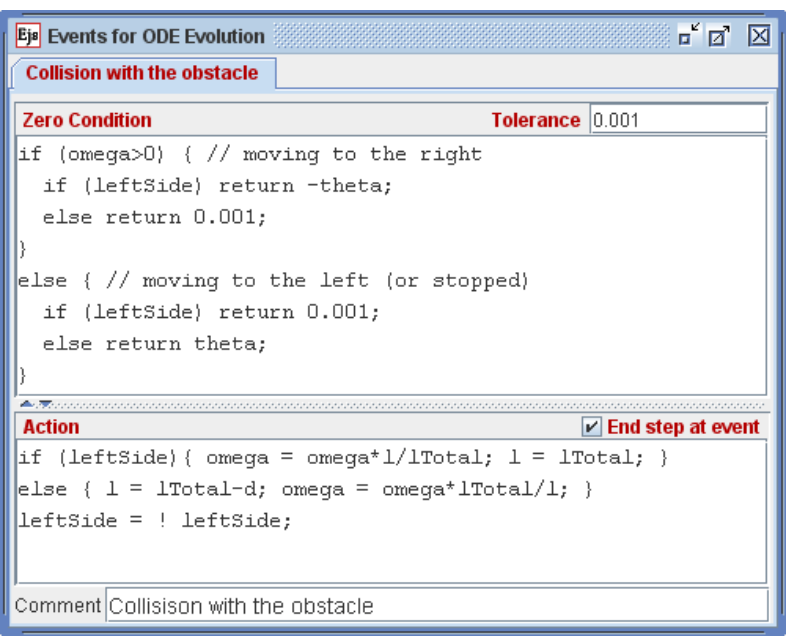

Notice how the collision computes the new angular velocity after the collision and adjust the value of $l$ to use.

\section{Constraints}

A page of constraints is required to compute the Cartesian coordinates from the angular ones.

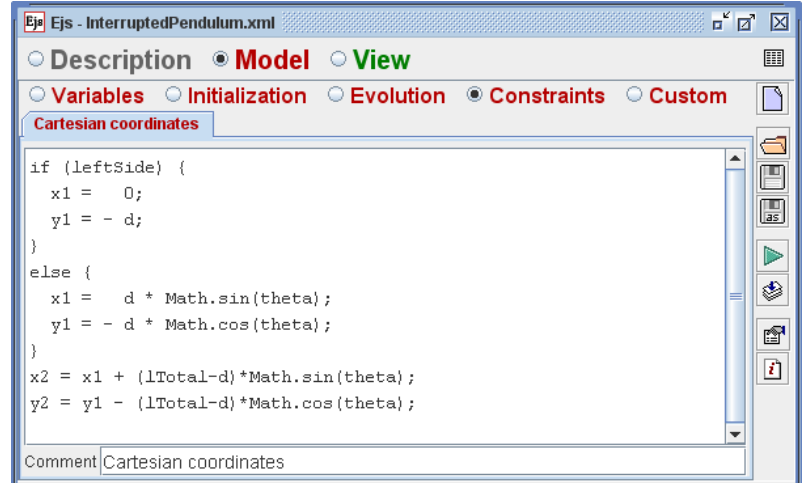

\section{Custom code}

No custom code required.

\section{View}

The view starts with the compound element based on a drawing panel with a default particle. We have added to the drawing panel a second particle to display the obstacle, and two strings (arrows with the Style property set to $S E G M E N T$ ) to complete the scene.

A plotting panel displayed in a separate Dialog window will plot the time evolution of the angle of the pendulum. (This is rather standard and we give no further details here.)

The complete Tree of Elements is displayed in the figure below:



For simplicity we have suppressed interaction with our view elements. The main properties that need to be modified are shown in the colo fields of the property panels below:

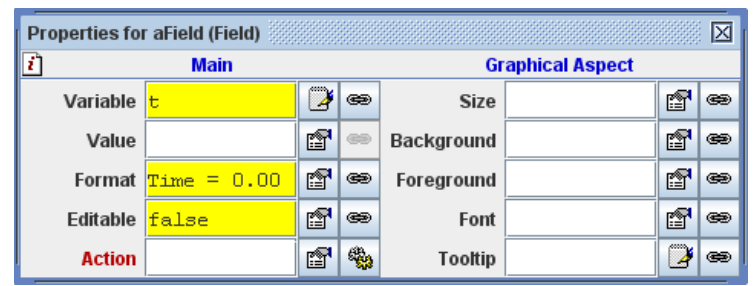




\section{Simple pendulum model in data warehouse for remlabnet}

Here we show the simple pendulum environment in RemLabNet day with output to data warehouse. Data are stored in OLAP cubes in the transaction concept store in the database. OLAP cubes are chosen due to the speed of analysis, which require immense computing power and access data stored on the measureserver. Data dimensional cubes to store our proposed method, where rows and columns are paired in three space and thus compare the cube to cube. Of course, the dice may be only 3 dimensional, but the number of dimensions is $\mathrm{X}$. For our work but sufficient dimension 3. Structure of tables and data is protected compartment of our system, that is not public. Outcomes data are of course fully public and the needs of users.

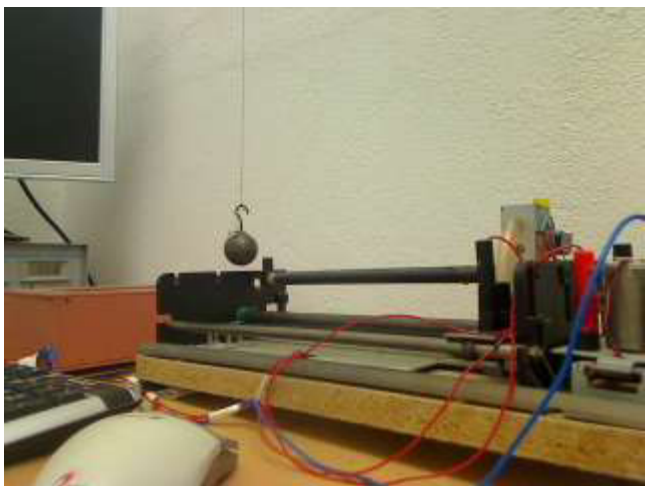

Figure E. RemLabnNet simple pendulum experiment.

public version of the data is as follows:

\begin{tabular}{|c|c|c|c|c|}
\hline Time $t[s]$ & Deviation & [rad] & Time $t$ [s] & Swing No. \\
\hline 1.351 & -0.042 & 1.351 & 0 & \\
\hline 1.371 & -0.075 & 1.371 & o & \\
\hline 1.391 & -0.095 & 1.391 & $\mathrm{o}$ & \\
\hline 1.411 & -0.087 & 1.411 & o & \\
\hline 1.431 & -0.058 & 1.431 & 1 & \\
\hline 1.451 & -0.053 & 1.451 & 1 & \\
\hline 1.471 & -0.066 & 1.471 & 1 & \\
\hline 1.491 & -0.088 & 1.491 & 1 & \\
\hline 1.511 & -0.077 & 1.511 & 2 & \\
\hline 1.531 & -0.061 & 1.531 & 2 & \\
\hline 1.551 & -0.056 & 1.551 & 2 & \\
\hline 1.570 & -0.068 & 1.570 & 2 & \\
\hline 1.591 & -0.071 & 1.591 & 3 & \\
\hline 1.611 & -0.058 & 1.611 & 3 & \\
\hline 1.631 & -0.053 & 1.631 & 3 & \\
\hline 1.650 & -0.060 & 1.650 & 3 & \\
\hline 1.671 & -0.069 & 1.671 & 4 & \\
\hline 1.691 & -0.060 & 1.691 & 4 & \\
\hline 1.711 & -0.051 & 1.711 & 4 & \\
\hline 1.730 & -0.054 & 1.730 & 4 & \\
\hline 1.751 & -0.058 & 1.751 & 4 & \\
\hline 1.771 & -0.059 & 1.771 & 5 & \\
\hline 1.791 & -0.059 & 1.791 & 5 & \\
\hline 1.811 & -0.057 & 1.811 & 5 & \\
\hline 1.831 & -0.052 & 1.831 & 6 & \\
\hline 1.851 & -0.053 & 1.851 & 6 & \\
\hline 1.871 & -0.060 & 1.871 & 6 & \\
\hline 1.891 & -0.064 & 1.891 & 6 & \\
\hline 1.911 & -0.053 & 1.911 & 7 & \\
\hline 1.931 & -0.045 & 1.931 & 7 & \\
\hline 1.951 & -0.055 & 1.951 & 7 & \\
\hline 1.971 & -0.060 & 1.971 & 7 & \\
\hline 1.991 & -0.054 & 1.991 & 8 & \\
\hline 2.011 & -0.046 & 2.011 & 8 & \\
\hline 2.031 & -0.049 & 2.031 & 8 & \\
\hline 2.051 & -0.054 & 2.051 & 9 & \\
\hline 2.071 & -0.056 & 2.071 & 9 & \\
\hline 2.091 & -0.050 & 2.091 & 9 & \\
\hline 2.111 & -0.049 & 2.111 & 9 & \\
\hline 2.131 & -0.049 & 2.131 & 10 & \\
\hline
\end{tabular}

Real running a simple pendulum experiment understandable model for the comparison of the simulations. Behavior, respectively, compared simulations and calculations from the actual operation is fascinating. So compare the article that focuses on the simulation of realistic simulation run. The picture is output in real measured data, which we can compare the result of the simulations. Measurement data in thousands of reported values is because of similar simulations, but in certain parts of the chart we see other shifts in behavior and real experiment.

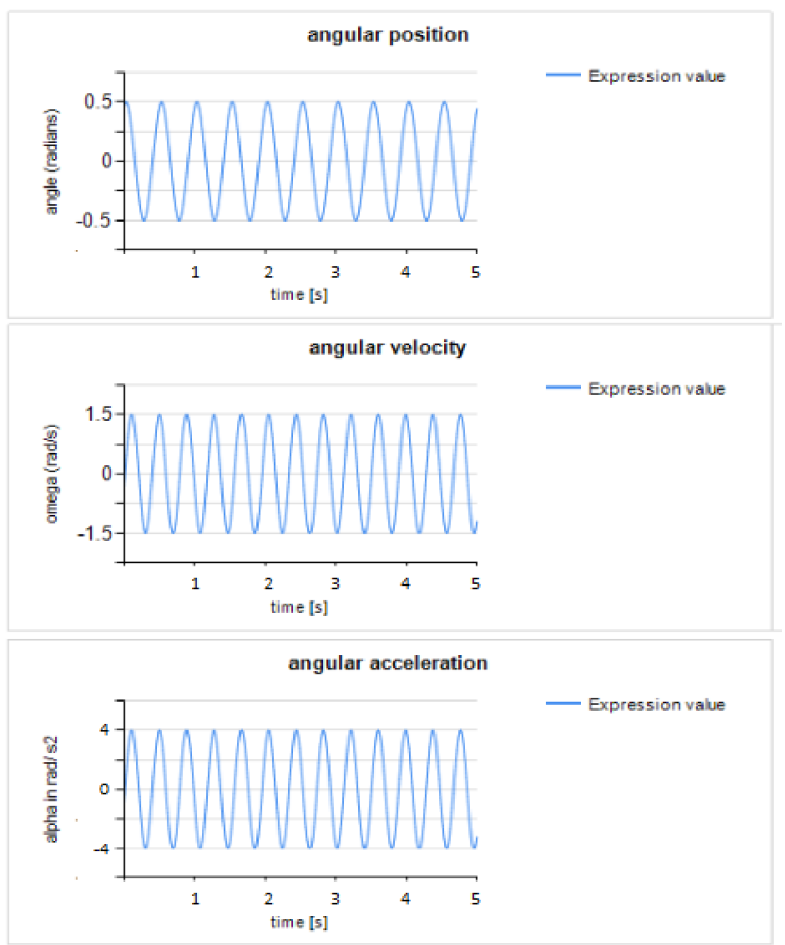

Figure F. graph of DW RemLabNet.

\section{Conclusions}

A simple pendulum mathematical model described above. It is a model of environmental RemLabNet, where the lessons we describe the theory and practice in simulation environments with remote experiments and analyzing the output data into the data warehouse RemLabNetu. From the article, it is obvious how the system works, all described by the theory, through calculation, via simulation to the real operation. Outputs are data from simulated and real environments from the measured data. Comparison of the results are fascinating. Great importance is being experinetů distant relative of both simulation and real factor systems running. In this article, however, we focus primarily on the simulation, which is described mathematically calculate all the characteristics and conditions and as such simulations in an environment easyjava simulation.

\section{Acknowledgments}

The paper was published thanks to the Grant of the Internal Agency of UTB No IGA/2016/. One of us acknowledges the partial support of the Slovak Research and Development Agency, project no. APVV-0096-11, the Scientific Grant Agency VEGA, project no. 
2/0157/12, and the KEGA Agency projects No 011TTU4/2012 and 020TTU-4/2013..

\section{References}

1. The whole system is detail described in the project proposal Submitted Project Grant Agency of the Czech Republic: INFORMATICS MEANS FOR GRID OF e-LABORATORIES - PROJECT REMLABNET, 2013.

2. KRBEČEK, Michal. Possible utilization of the artificial intelligence elements in the creation of remote experiments. [online]. 2012, č. 1 [cit. 201306-26]

3. Grid Remote Laboratory Management System. Sahara Reaches Europe. 2013, č. 1

4. Database-Level Roles [online]. 2012 [cit. 2013-0626], http://msdn.microsoft.com/enus/library/ms189121.aspx

5. ALEXANDER, David, Amanda FINCH, David SUTTON a Andy TAYLOR. Information Security Management Principles. 2. vyd. bcs, 2013. ISBN 9781780171753

6. SCHULZ. Cloud and Virtual Data Storage Networking. teChapterChapterbooks, 2011. ISBN 978-1439851739

7. Data Warehouse [online]. 2013 [cit. 2013-06-26], http://en.wikipedia.org/wiki/Data_warehouse

8. SCHAUER, František, František LUSTIG a Miroslava OŽVOLDOVÁ. Innovations 2011: World Innovations in Engineering Education and Research: Internet Natural Science Remote e-Laboratory (INTRE-L) for Remote Experiments. USA: iNEER, 2011, s. 51-68. 1. ISBN 978-0-9818868-2-4.

9. SCHAUER, František a Miroslava OŽVOLDOVÁ. Plug and play system for hands on and remote laboratories. In: Proceedings of 8th International Conference on Hands-on Science. Ljubljana: University of Ljubljana, 2011, s. 17-21. ISBN 978989-95095-7-3

10. KRBEČEK Michal, František SCHAUER, Roman JAŠEK. Security aspects of remote e-laboratories. Zlín: UTB ve Zlíně, Fakulta aplikované informatiky, 2012

11. PÁLKA Lukáš,Data Warehouse services [online]. 2013 [cit. 2013-06-26], http://datawarehouse.cz/Data_warehouse

12. Data Warehouse [online]. 2013 [cit. 2013-06-25], http://www.1 keydata.com/datawarehousing/dataware house.html

13. PÁLKA Lukáš, Methods and Tools Related to Data Security and the Protection of Microsoft SQL Servers. Zlín UTB, 2012

14. LABERGE, Robert. The Data Warehouse Mentor: Practical Data Warehouse and Business Intelligence Insights. -: 2011. ISBN-10: 0071745327

15. CLARKE, Justin. SQL Injection Attacks and Defense. USA: Elsevier, 2012. ISBN 978-1-59749963-7.

16. GERŽA Michal, František SCHAUER, Roman JAŠEK. Security of ISES MeasureServer` module for remote experiments against malign attacks, Zlín: UTB ve Zlíně, Fakulta aplikované informatiky, 2013.

17. BRUCHEZ, Rudi. Microsoft SQL Server 2012 Security Cookbook. UK: Packt Publishing, 2012. ISBN ISBN 978-1-84968-588-7

18. HARKINS, Malcolm. Managing Risk and Information Security: Protect to Enable. LLC: Apress Media, 2013. ISBN 978-1430251132

19. Paper REV 2014 - 31102013 - F. Schauer 2014

20. T. Dulík, M. Bližňák, "Security measures in virtual laboratory of microprocessor technology," DAAAM International Vienna, Proceedings of the 21st International DAAAM Symposium "Intelligent Manufacturing \& Automation: Focus on Interdisciplinary Solutions", Vienna, 2010, pp. 12031204, ISBNISSN 978-3-901509-73-5

21. D. Lowe, P, Newcombe and B. Stumpers, "Evaluation of the Use of Remote Laboratories for Secondary," Res. Sci. Educ/Science Education, Springer Science and Business Media B.V. 2012, DOI 10.1007/s11165-012-9304-3.

22. Lustig, F.: "Počítačem podporované školní experimenty $\mathrm{s}$ měřícím systémem ISES pod Windows", In: sborník MEDACTA 97 - vzdělávanie v meniacom sa svete, 404-408, Ústav didaktickej technológie, PF UKF v Nitre, Nitra, ISBN 80967339-9-0, 1997 and Lustig, F. and Schauer, F.: "Creative laboratory experiments for basic physics using computer data collection and evaluation exemplified on the ISES", Proceedings first european conference on Physics Teaching in Engineering Education, 125-131, Copenhagen, Denmark, ed. Oehlenschlaeger, 1997 